\title{
FURTHER INVESTIGATIONS WITH THE STRONG PROBABLE PRIME TEST
}

\author{
RONALD JOSEPH BURTHE, JR.
}

\begin{abstract}
Recently, Damgård, Landrock and Pomerance described a procedure in which a $k$-bit odd number is chosen at random and subjected to $t$ random strong probable prime tests. If the chosen number passes all $t$ tests, then the procedure will return that number; otherwise, another $k$-bit odd integer is selected and then tested. The procedure ends when a number that passes all $t$ tests is found. Let $p_{k, t}$ denote the probability that such a number is composite. The authors above have shown that $p_{k, t} \leq 4^{-t}$ when $k \geq 51$ and $t \geq 1$. In this paper we will show that this is in fact valid for all $k \geq 2$ and $t \geq 1$.
\end{abstract}

\section{INTRODUCTION}

Let $n$ be an odd number with $n-1=2^{s} u$, where $u$ is odd. The following notation will be used in this article:

$\mathcal{S}(n)=\left\{a \in[1, n-1]: a^{u} \equiv 1 \bmod n\right.$ or $a^{2^{i} u} \equiv-1 \bmod n$ for some $i=0,1, \ldots$, $s-1\}$,

$S(n)=|\mathcal{S}(n)|$.

If $a \in \mathcal{S}(n)$ for some pair $a$ and $n$, we say that $n$ is a strong probable prime to base $a$. If $n$ is prime, then $S(n)=n-1$, and if $n$ is an odd composite number, then $S(n) /(n-1) \leq 1 / 4$ (see Monier [4], Rabin [5]).

Now if for a given $n$ we can find an integer $a \in[1, n-1]$ such that $a \notin \mathcal{S}(n)$, then we know that $n$ is composite. If one picks $t a$ 's at random from $[1, n-1]$ and discovers that each is in $\mathcal{S}(n)$, one cannot however conclude that $n$ is prime. We can conclude that if $n$ is an odd composite number, the probability that all the $t$ randomly chosen $a$ 's are in $\mathcal{S}(n)$ is less than or equal to $4^{-t}$.

These results suggest a procedure for finding random integers that are likely to be prime in the set $M_{k}$ of odd $k$-bit integers. Choose a random $n$ in $M_{k}$ and then choose an $a_{1} \in[1, n-1]$ and see if $a_{1} \in \mathcal{S}(n)$. If $a_{1} \in \mathcal{S}(n)$, then choose an $a_{2} \in[1, n-1]$ and test to see if it is in $\mathcal{S}(n)$. This procedure is then repeated until either an $a_{i}$ is discovered such that $a_{i} \notin \mathcal{S}(n)$ or until $t a_{i}$ 's are found that are all in $\mathcal{S}(n)$. In the former case, another $n$ is picked from $M_{k}$, and in the latter case, the number $n$ will be given as output. This procedure, as described in [1] will be referred to here as the random bases procedure.

Let $p_{k, t}$ denote the probability that the number which is given as output by the random bases procedure is composite. In [2], it is left as an open question to find a value $k_{0}$ such that $p_{k, t} \leq 4^{-t}$ for all $t \geq 1$ and $k \geq k_{0}$. From Monier's and Rabin's

Received by the editor May 3, 1994.

1991 Mathematics Subject Classification. Primary 11Y11; Secondary 11A51. 
result one sees that if $n$ is an odd composite integer, then the probability that it passes $t$ random strong pseudoprime tests is less than or equal to $4^{-t}$. However, this is not sufficient to show that $p_{k, t} \leq 4^{-t}$ as the following discussion shows. For a fixed $t \geq 1$ choose $k$ sufficiently large such that the density of the primes in $M_{k}$ is much less than $4^{-t}$. Assume also that for most composite $m$ in $M_{k}$ that the probability that $m$ passes a random bases test is about $1 / 4$. Then, of course, the probability of it passing $t$ tests is about $4^{-t}$. Suppose that we have an $n$ from $M_{k}$ that passes $t$ tests. Since we are assuming that the primes in $M_{k}$ are scarce, it will be much more likely that $n$ is composite rather than prime. So $p_{k, t}$ would be close to 1 . However, it will be shown in this dissertation, that $p_{k, t} \leq 4^{-t}$ for $k \geq 2$ and $t \geq 1$. The flawed assumption that led us to the conclusion that $p_{k, t}$ was close to 1 is the assumption that the probability of a composite $n$ in $M_{k}$ passing a test was about $1 / 4$. In actuality the probability is usually much smaller and this is essentially the conclusion of Proposition 1.

In the next section we will prove that $p_{k, t} \leq(1 / 4)^{t-l} p_{k, l} /\left(1-p_{k, l}\right)$ for integer $l$ with $1 \leq l \leq t-1$. Taking $l=1$ we get that $p_{k, t} \leq 4^{1-t} p_{k, 1} /\left(1-p_{k, 1}\right)$, so to show that $p_{k, t} \leq 4^{-t}$ for all $t \geq 1$, it suffices to show that $p_{k, 1} \leq 1 / 5$. In [3], it is shown that this is true for $k \geq 55$. Taking $l=2$ in the above inequality, we can see that to show that $p_{k, t} \leq 4^{-t}$ for all $t \geq 1$ it will also suffice to show that $p_{k, 1} \leq 1 / 4$ and $p_{k, 2} \leq 1 / 17$. In [3], this is shown to be true for all $k$ with $51 \leq k \leq 54$. In this paper we will improve the results in [3] and show that $p_{k, t} \leq 4^{-t}$ for all $k$ with $k \geq 2$ and $t \geq 1$. This will be done by extending some of the ideas in [3] and sharpening the upper bounds found there as well. Some improvements are due to simple observations of the properties of certain numbers and easily lead to a lower upper bound. Other improvements are not quite as obvious and require more work while only minimally improving some of the results. The overall net effect is to reduce in general the upper bound for $p_{k, t}$ by a factor of a fourth. We are able to prove a theorem that enabled us to verify that $p_{k, t} \leq 4^{-t}$ for all $k \geq 25$ and $t \geq 1$. For $2 \leq k \leq 24$, the result is verified by actually computing $p_{k, t}$ using an equation due to Monier. Thus we can take $k_{0}$ to be 2 .

\section{Preliminaries}

We will start by recalling Lemma 1 from [3]. Here, $\omega(n)$ is the number of distinct prime factors of $n, \Omega(n)$ is the number of prime factors of $n$ counted with multiplicity, $\phi(n)$ is the Euler phi function, and $\alpha(n)=S(n) / \phi(n)$. For the remainder of the paper, $p$ will always be used to denote a prime.

Lemma 1. If $n>1$ is odd, then

$$
\frac{1}{\alpha(n)} \geq 2^{\omega(n)-1} \prod_{p^{\beta} \|_{n}} p^{\beta-1} \frac{p-1}{(p-1, n-1)} \geq 2^{\Omega(n)-1} \prod_{p \mid n} \frac{p-1}{(p-1, n-1)}
$$

The following lemma is a generalization of Lemma 2 in [3] and gives a slightly improved result.

Lemma 2. If $t \in \mathbb{R}, t \geq s, s \in \mathbb{Z}^{+}$, then

$$
\sum_{n=\lfloor t\rfloor+1}^{\infty} \frac{1}{n^{2}}<\frac{c_{s}}{t}
$$


where

$$
c_{s}=(s+1)\left(\frac{\pi^{2}}{6}-\sum_{n=1}^{s} \frac{1}{n^{2}}\right)
$$

Proof. Let $m=\lfloor t\rfloor$. So $m \in \mathbb{Z}, m \geq s$. Then

$$
\begin{aligned}
\sum_{n=m+1}^{\infty} \frac{1}{n^{2}} & =\sum_{n=1}^{\infty} \frac{1}{n^{2}}-\sum_{n=1}^{m} \frac{1}{n^{2}}=\frac{\pi^{2}}{6}-\sum_{n=1}^{m} \frac{1}{n^{2}} \\
& <\frac{(m+1)}{t}\left(\frac{\pi^{2}}{6}-\sum_{n=1}^{m} \frac{1}{n^{2}}\right)=\frac{1}{t} c_{m} .
\end{aligned}
$$

Letting $k \in \mathbb{Z}^{+}$with $k \geq s+1$, we have that

$$
\begin{aligned}
c_{k-1}-c_{k} & =-\frac{\pi^{2}}{6}+\frac{1}{k}+\sum_{n=1}^{k} \frac{1}{n^{2}} \\
& =-\frac{\pi^{2}}{6}+\int_{k}^{\infty} \frac{1}{x^{2}} d x+\sum_{n=1}^{k} \frac{1}{n^{2}}>-\frac{\pi^{2}}{6}+\sum_{n=1}^{\infty} \frac{1}{n^{2}}=0 .
\end{aligned}
$$

Thus the sequence $c_{s}, c_{s+1}, \ldots$ is decreasing, and in particular $c_{m} \leq c_{s}$. Substituting into the previous inequality gives the desired result.

Lemma 3. If $l, t \in \mathbb{Z}^{+}$with $1 \leq l \leq t-1$, then

$$
p_{k, t} \leq 4^{-l} \frac{p_{k, l}}{1-p_{k, l}} .
$$

Proof. The event that a number chosen at random from $M_{k}$ passes the $i$ th test will be denoted by $D_{i}$, and we define the event $E_{i}$ by

$$
E_{i}=D_{1} \cap D_{2} \cap \cdots \cap D_{i} .
$$

We will also let $C$ denote the event that a number chosen at random from $M_{k}$ is composite, and $C^{\prime}$ will denote the set of composites. Also let $\bar{\alpha}(n)=S(n) /(n-1)$, and recall that for odd composite $n$ we will have $\bar{\alpha}(n) \leq 1 / 4 . \quad P(A)$ will be used here to denote the probability that event $A$ occurs. Note that $P\left(C \cap E_{i}\right)=$ $2^{-(k-2)} \sum_{n \in C^{\prime} \cap M_{k}} \bar{\alpha}(n)^{i}$.

Now for $1 \leq l \leq t-1$ we have

$$
\begin{aligned}
p_{k, t} & =P\left(C \mid E_{t}\right)=\frac{P\left(C \cap E_{t}\right)}{P\left(E_{t}\right)} \\
& =\frac{P\left(C \cap E_{t}\right)}{P\left(C \cap E_{t-1}\right)} \frac{P\left(C \cap E_{t-1}\right)}{P\left(C \cap E_{t-2}\right)} \ldots \frac{P\left(C \cap E_{l+1}\right)}{P\left(C \cap E_{l}\right)} \frac{P\left(C \cap E_{l}\right)}{P\left(E_{t}\right)} .
\end{aligned}
$$

Now

$$
\frac{P\left(C \cap E_{i}\right)}{P\left(C \cap E_{i-1}\right)}=\frac{\sum_{n \in C^{\prime} \cap M_{k}} \bar{\alpha}(n)^{i}}{\sum_{n \in C^{\prime} \cap M_{k}} \bar{\alpha}(n)^{i-1}} \leq \frac{\sum_{n \in C^{\prime} \cap M_{k}} \frac{1}{4} \bar{\alpha}(n)^{i-1}}{\sum_{n \in C^{\prime} \cap M_{k}} \bar{\alpha}(n)^{i-1}}=\frac{1}{4} .
$$


Letting $A^{c}$ denote the complement of event $A$, we see that $C^{c}$ is the event that a number is prime. Since a prime in $M_{k}$ will always pass each test we see that $P\left(C^{c} \cap E_{t}\right)=P\left(C^{c}\right)=P\left(C^{c} \cap E_{l}\right)$. Thus we see that

$$
p_{k, t} \leq\left(\frac{1}{4}\right)^{t-l} \frac{P\left(C \cap E_{l}\right)}{P\left(E_{l}\right)} \frac{P\left(E_{l}\right)}{P\left(E_{t}\right)}=\left(\frac{1}{4}\right)^{t-l} p_{k, l} \frac{P\left(E_{l}\right)}{P\left(E_{t}\right)}
$$

Also

$$
\frac{P\left(E_{l}\right)}{P\left(E_{t}\right)} \leq \frac{P\left(E_{l}\right)}{P\left(C^{c} \cap E_{t}\right)}=\frac{P\left(E_{l}\right)}{P\left(C^{c}\right)}=\frac{P\left(E_{l}\right)}{P\left(C^{c} \cap E_{l}\right)}=\frac{1}{P\left(C^{c} \mid E_{l}\right)}=\frac{1}{1-p_{k, l}}
$$

which completes the proof of the lemma.

\section{Estimates}

Now as in [3], $C_{m}$ will denote the set of odd composite integers $n$ with $\alpha(n)>$ $2^{-m}$. However, we will allow $m$ to assume nonintegral values. Since $\alpha(n) \leq 1 / 4$ for odd composite $n \neq 9$ (see [4] or [5]) and since $\alpha(9)=1 / 3$, we will have $C_{m}=\emptyset$ for $0<m \leq \ln 3 / \ln 2$ and $C_{m}=\{9\}$ for $\ln 3 / \ln 2<m \leq 2$.

We will now generalize Theorem 1 from [3] for the case where $m$ is not necessarily an integer.

Theorem 1. Assume $k \in \mathbb{Z}^{+}, k \geq 2, m \in \Re^{+}, s \in \mathbb{Z}^{+}$such that

$$
s \leq\left(2^{(k-1) / j}-1\right) 2^{j-m-2} \quad \text { for } j=2,3, \ldots,\lceil m\rceil .
$$

Then

$$
\frac{\left|C_{m} \cap M_{k}\right|}{\left|M_{k}\right|}<2 c_{s} \sum_{j=2}^{\lceil m\rceil} \frac{\left\lceil 2^{m+1-j}\right\rceil-1}{2^{\frac{k-1}{j}}-1}
$$

where $c_{s}$ is defined as in Lemma 2.

Proof. From Lemma 1, we have $1 / \alpha(n) \geq 2^{\Omega(n)-1}$ for all odd $n$. So if $n \in C_{m}$, we have $2^{m}>1 / \alpha(n) \geq 2^{\Omega(n)-1}$ and thus $m+1>\Omega(n)$. Since $\Omega(n) \in \mathbb{Z}^{+}$, this implies that $\Omega(n) \leq\lceil m\rceil$. Now letting $N(m, k, j)=\left\{n \in C_{m} \cap M_{k} \mid \Omega(n)=j\right\}$, we see that

$$
\left|C_{m} \cap M_{k}\right|=\sum_{j=2}^{\lceil m\rceil}|N(m, k, j)|
$$

Let $n \in N(m, k, j), 2 \leq j \leq\lceil m\rceil$, and let $p$ be the largest prime factor of $n$. Now $2^{k-1}<n \leq p^{j}$ implies that $p>2^{(k-1) / j}$. Let $d(p, n)=(p-1) /(p-1, n-1)$. Lemma 1 implies that $2^{m}>\alpha(n)^{-1} \geq 2^{\Omega(n)-1} d(p, n)=2^{j-1} d(p, n)$, so we must have $d(p, n)<2^{m+1-j}$.

Given $p, d$ such that $p>2^{(k-1) / j}, d \mid p-1$, and $d<2^{m+1-j}$, we want to get an upper bound for the number of $n \in N(m, k, j)$ with largest prime factor $p$ and $d(p, n)=d$; it will suffice to consider the set $S_{k, d, p}:=\left\{n \in M_{k}: p \mid n, d=d(p, n)\right.$, $n$ composite $\}$. The set $S_{k, d, p}$ is contained in the set $R_{k, d, p}:=\{n \in \mathbb{Z}: n \equiv 0 \bmod p$, $\left.n \equiv 1 \bmod (p-1) / d, p<n<2^{k}\right\}$ which has, via the Chinese Remainder Theorem, 
less than $2^{k} d /(p(p-1))$ elements. If $S_{k, d, p} \neq \emptyset$, then there exists an $n \in S_{k, d, p}$ with $(n-1, p-1)=(p-1) / d$, and thus $(p-1) / d$ must be even since $n$ and $p$ are odd. Thus we need only consider those $d$ and $p$ such that $(p-1) / d$ is even. Letting $\sum^{o}$ denote a sum over $p$ with $2^{\frac{k-1}{j}}<p<2^{k}, d \mid p-1$ and $(p-1) / d$ even, we get that

$$
\begin{aligned}
|N(m, k, j)| & \leq \sum_{d<2^{m+1-j}} \sum^{o}\left|S_{k, d, p}\right| \leq \sum_{d<2^{m+1-j}} \sum^{o}\left|R_{k, d, p}\right| \\
& \leq 2^{k} \sum_{d<2^{m+1-j}} \sum^{o} \frac{d}{p(p-1)} .
\end{aligned}
$$

Now

$$
\begin{aligned}
\sum^{o} \frac{d}{p(p-1)} & \leq \sum_{2 u d>2^{(k-1) / j}-1} \frac{d}{(2 u d+1) 2 u d} \leq \frac{1}{4 d} \sum_{u>\frac{2^{(k-1) / j}-1}{2 d}} \frac{1}{u^{2}} \\
& <\frac{1}{4 d} \frac{2 c_{s} d}{\left(2^{(k-1) / j}-1\right)}=\frac{c_{s}}{2} \frac{1}{\left(2^{(k-1) / j}-1\right)}
\end{aligned}
$$

with the last inequality coming from Lemma 2. Note: to use Lemma 2, we must have that $\left(2^{(k-1) / j}-1\right) /(2 d) \geq s$, but this follows from the hypothesis that $s \leq$ $\left(2^{(k-1) / j}-1\right) 2^{j-m-2}$, since $d<2^{m+1-j}$. Thus,

$$
|N(m, k, j)| \leq 2^{k} \frac{c_{s}}{2} \sum_{d<2^{m+1-j}} \frac{1}{2^{(k-1) / j}-1}=2^{k} \frac{c_{s}}{2} \frac{\left\lceil 2^{m+1-j}\right\rceil-1}{2^{(k-1) / j}-1} .
$$

Therefore,

$$
\left|C_{m} \cap M_{k}\right|=\sum_{j=2}^{\lceil m\rceil}|N(m, k, j)|<2^{k} \frac{c_{s}}{2} \sum_{j=2}^{\lceil m\rceil} \frac{\left\lceil 2^{m+1-j}\right\rceil-1}{2^{(k-1) / j}-1} .
$$

Now since $k \geq 2$, we have $\left|M_{k}\right|=2^{k-2}$. So by dividing each side of the inequality by $2^{k-2}$ we get the desired result and our proof of Theorem 1 is complete.

Now let $\sum^{\prime}$ denote a sum over composite integers. As in [3], recalling that $p$ denotes a prime, we have

$$
\begin{aligned}
p_{k, t} & =\left(\sum_{n \in M_{k}} \bar{\alpha}(n)^{t}\right)^{-1} \sum_{n \in M_{k}}^{\prime} \bar{\alpha}(n)^{t}=\left(\sum_{n \in M_{k}}^{\prime} \bar{\alpha}(n)^{t}+\sum_{p \in M_{k}} 1\right)^{-1} \sum_{n \in M_{k}}^{\prime} \bar{\alpha}(n)^{t} \\
& =\left(\sum_{n \in M_{k}}^{\prime} \bar{\alpha}(n)^{t}+\pi\left(2^{k}\right)-\pi\left(2^{k-1}\right)\right)^{-1} \sum_{n \in M_{k}}^{\prime} \bar{\alpha}(n)^{t} .
\end{aligned}
$$

Now if we have an upper bound $N_{1}$ for $\sum_{n \in M_{k}}^{\prime} \bar{\alpha}(n)^{t}$ and a lower bound $P_{1}$ for $\pi\left(2^{k}\right)-\pi\left(2^{k-1}\right)$, then

$$
p_{k, t} \leq \frac{N_{1}}{N_{1}+P_{1}}
$$

Let $\sum^{(q)}$ denote a sum with increments of length $1 / q$ where $q \in \mathbb{Z}^{+}$. 
Proposition 1. Let $k, M, t, s, q \in \mathbb{Z}^{+}$with $k \geq 5, M \geq 3$ and $s \leq$ $\left(2^{(k-1) / j}-1\right) 2^{j-M-2}$ for $j=2,3, \ldots, M$. Then

$$
\sum_{n \in M_{k}}^{\prime} \bar{\alpha}(n)^{t} \leq 2^{-M t+k-2}+2^{k-1} c_{s}\left(2^{t / q}-1\right) \sum_{m=2+\frac{1}{q}}^{M} 2^{-m t} \sum_{j=2}^{\lceil m\rceil} \frac{\left\lceil 2^{m+1-j}\right\rceil-1}{2^{\frac{k-1}{j}}-1} .
$$

Proof. Since $k \geq 5$, we have $9 \notin M_{k}$. So for all $m$ with $0<m \leq 2$, we have $C_{m} \cap M_{k}=\emptyset$. Thus

$$
\begin{aligned}
& \sum_{n \in M_{k}}^{\prime} \bar{\alpha}(n)^{t}=\sum_{m=2+\frac{1}{q}}^{\infty}(q) \sum_{n \in M_{k} \cap\left(C_{m} \backslash C_{m-\frac{1}{q}}\right)} \bar{\alpha}(n)^{t} \leq \sum_{m=2+\frac{1}{q}}^{\infty}(q) \sum_{n \in M_{k} \cap\left(C_{m} \backslash C_{m-\frac{1}{q}}\right)} \alpha(n)^{t} \\
& \quad \leq \sum_{m=2+\frac{1}{q}}^{\infty} 2^{-\left(m-\frac{1}{q}\right) t}\left|M_{k} \cap\left(C_{m} \backslash C_{m-\frac{1}{q}}\right)\right| \\
& \leq 2^{-M t} \sum_{m=M+\frac{1}{q}}^{\infty}\left|M_{k} \cap\left(C_{m} \backslash C_{m-\frac{1}{q}}\right)\right|+\sum_{m=2+\frac{1}{q}}^{(q)} 2^{-\left(m-\frac{1}{q}\right) t}\left|M_{k} \cap\left(C_{m} \backslash C_{m-\frac{1}{q}}\right)\right| \\
& =2^{-M t}\left|M_{k} \backslash C_{M}\right|+\sum_{m=2+\frac{1}{q}}^{M} 2^{-\left(m-\frac{1}{q}\right) t}\left|M_{k} \cap\left(C_{m} \backslash C_{m-\frac{1}{q}}\right)\right| .
\end{aligned}
$$

Let $T_{m}=\left|C_{m} \cap M_{k}\right|$, and let $U_{m}$ be an upper bound for $T_{m}$. Rewriting the above inequality, we get

$$
\begin{aligned}
& \sum_{n \in M_{k}}^{\prime} \bar{\alpha}(n)^{t} \leq 2^{-M t}\left(\left|M_{k}\right|-T_{M}\right)+\sum_{m=2+\frac{1}{q}}^{M} 2^{-\left(m-\frac{1}{q}\right) t}\left(T_{m}-T_{m-\frac{1}{q}}\right) \\
& =2^{-M t}\left(2^{k-2}-T_{M}\right)+\sum_{m=2+\frac{1}{q}}^{M-\frac{1}{q}}\left(2^{-\left(m-\frac{1}{q}\right) t}-2^{-m t}\right) T_{m}+2^{-\left(M-\frac{1}{q}\right) t} T_{M}-2^{-2 t} T_{2} \\
& \leq 2^{-M t+k-2}+\sum_{m=2+\frac{1}{q}}^{M}\left(2^{-\left(m-\frac{1}{q}\right) t}-2^{-m t}\right) T_{m} \\
& \leq 2^{-M t+k-2}+\sum_{m=2+\frac{1}{q}}^{M}\left(2^{-\left(m-\frac{1}{q}\right) t}-2^{-m t}\right) U_{m} .
\end{aligned}
$$

Now $2^{-\left(m-\frac{1}{q}\right) t}-2^{-m t}=\left(2^{\frac{t}{q}}-1\right) 2^{-m t}$, and since we can take

$$
U_{m}=2^{k-1} c_{s} \sum_{j=2}^{\lceil m\rceil} \frac{\left\lceil 2^{m+1-j}\right\rceil-1}{2^{\frac{k-1}{j}}-1}
$$

from Theorem 1, this gives 


$$
\sum_{n \in M_{k}}^{\prime} \bar{\alpha}(n)^{t} \leq 2^{-M t+k-2}+2^{k-1} c_{s}\left(2^{\frac{t}{q}}-1\right) \sum_{m=2+\frac{1}{q}}^{M}(q) 2^{-m t} \sum_{j=2}^{\lceil m\rceil} \frac{\left\lceil 2^{m+1-j}\right\rceil-1}{2^{\frac{k-1}{j}}-1} .
$$

This concludes our proof of Proposition 1.

From Proposition 2 in [3], we know that for $k \geq 21$ we have

$$
\pi\left(2^{k}\right)-\pi\left(2^{k-1}\right)>(.71867) \frac{2^{k}}{k} .
$$

Thus, we may take $N_{1}$ and $P_{1}$ in (1) as

$$
N_{1}=2^{-M t+k-2}+2^{k-1} c_{s}\left(2^{\frac{t}{q}}-1\right) \sum_{m=2+\frac{1}{q}}^{M}(q) 2^{-m t} \sum_{j=2}^{\lceil m\rceil}\left(\left\lceil 2^{m+1-j}\right\rceil-1\right) /\left(2^{\frac{k-1}{j}}-1\right)
$$

and

$$
P_{1}=(.71867) \frac{2^{k}}{k}
$$

for $k \geq 21$, where $M \geq 3$ and $s \leq\left(2^{(k-1) / j}-1\right) 2^{j-M-2}$ for $2 \leq j \leq M$.

Now in our computations we need $s$ to be a positive integer at most $\left(2^{(k-1) / j}-1\right) 2^{j-M-2}$ for each $j$ with $2 \leq j \leq M$. So we choose $M$ such that the greatest integer less than or equal to $\left(2^{(k-1) / j}-1\right) 2^{j-M-2}$ is positive. So essentially we want to have $g(j):=(k-1) / j+j-M-2 \geq 1$. Taking $M$ and $k$ as fixed, we see that $g$ has a minimum of $-M-2+2 \sqrt{k-1}$ at $j=\sqrt{k-1}$. So for our purposes, it is sufficient to choose $M$ such that $3 \leq M \leq 2 \sqrt{k-1}-3$. This guarantees that we can find a largest positive integer - say $s_{1}$ - with $s_{1} \leq$ $\left(2^{(k-1) / j}-1\right) 2^{j-M-2}$ for each $j$ with $2 \leq j \leq M$. Choosing $s=s_{1}$ gives us the best possible constant for $c_{s}$ (but computing $c_{s_{1}}$ for large $s_{1}$ uses more running time while yielding only negligible improvements). So for each $M$ with $3 \leq M \leq 2 \sqrt{k-1}-3$, we choose $s$ to be the minimum of $s_{1}$ and 30 and compute our upper bound using that $s$ and taking $q=4$. Taking the minimum of all the upper bounds we were able to show that $p_{k, 1} \leq 1 / 4$ and $p_{k, 2} \leq 1 / 17$ for $25 \leq k \leq 50$. Thus we were able to show that $p_{k, t} \leq 4^{-t}$ for all $k$ with $25 \leq k \leq 50$. In our approximations for the upper bound of $p_{k, t}$, the $s$-value we chose was only dependent upon $M$ but if we brought the $c_{s}$ inside the summation over $m$ in the above expression for $N_{1}$, we could have in fact chosen our $s$ so that it would be dependent upon $m$ instead, and it would only have to satisfy the inequality $s \leq\left(2^{(k-1) / j}-1\right) 2^{j-m-2}$. This was attempted for some values of $k$ but it did not produce any significant improvements. Subsequently, we continued to use the value for $s$ determined by $M$. The main results of our computations are shown in Table I and Table II.

TABLE I. $u_{k}$ : upper bound for $p_{k, 1}$ for $25 \leq k \leq 50$

\begin{tabular}{c|c||c|c||c|c||c|c}
$k$ & $u_{k}$ & $k$ & $u_{k}$ & $k$ & $u_{k}$ & $k$ & $u_{k}$ \\
\hline 25 & 0.248692 & 32 & 0.173705 & 39 & 0.119921 & 46 & 0.080952 \\
26 & 0.237095 & 33 & 0.165640 & 40 & 0.113131 & 47 & 0.076863 \\
27 & 0.225027 & 34 & 0.157518 & 41 & 0.107492 & 48 & 0.072736 \\
28 & 0.215733 & 35 & 0.150928 & 42 & 0.102743 & 49 & 0.069210 \\
29 & 0.209292 & 36 & 0.144473 & 43 & 0.099016 & 50 & 0.066400 \\
30 & 0.196233 & 37 & 0.135932 & 44 & 0.091629 & & \\
31 & 0.185387 & 38 & 0.126492 & 45 & 0.086431 & &
\end{tabular}


TABLE II. $u_{k, 2}$ : upper bound for $p_{k, 2}$ for $25 \leq k \leq 50$

\begin{tabular}{l|c||l|l||l|l||l|l}
$k$ & $u_{k, 2}$ & $k$ & $u_{k, 2}$ & $k$ & $u_{k, 2}$ & $k$ & $u_{k, 2}$ \\
\hline 25 & .021590 & 32 & .008395 & 39 & .003472 & 46 & .001476 \\
26 & .018884 & 33 & .007394 & 40 & .003027 & 47 & .001315 \\
27 & .016233 & 34 & .006436 & 41 & .002659 & 48 & .001160 \\
28 & .014140 & 35 & .005650 & 42 & .002349 & 49 & .001029 \\
29 & .012564 & 36 & .004996 & 43 & .002099 & 50 & .000922 \\
30 & .011127 & 37 & .004471 & 44 & .001869 & & \\
31 & .009791 & 38 & .003928 & 45 & .001672 & &
\end{tabular}

\section{Exact values}

The above upper bound method fails to give us the desired result for $k \leq 24$ and in this section the method used to handle these cases will be discussed. Let $\nu(n)$ denote the largest integer such that $2^{\nu(n)} \mid p-1$ for each prime $p \mid n$, and let $u$ denote the largest odd factor of $n-1$. If one recalls that $\bar{\alpha}(n)=S(n) /(n-1)$, it is computationally possible to compute $p_{k, 1}$ exactly for $k \leq 24$, using (as described in [4]) Monier's result

$$
S(n)=\left(1+\frac{2^{\omega(n) \nu(n)}-1}{2^{\omega(n)}-1}\right) \prod_{p \mid n}(p-1, u)
$$

and our previous formula (recalling that $\sum^{\prime}$ is a sum over composite integers)

$$
p_{k, 1}=\left(\sum_{n \in M_{k}} \bar{\alpha}(n)\right)^{-1} \sum_{n \in M_{k}}^{\prime} \bar{\alpha}(n) .
$$

To compute $S(n)$, all of the prime factors of $n$ must be determined so computationally we can only use this formula for fairly small values of $k$. The $\mathrm{C}$ program we used for our computations first found all the primes less than 4096 (the square root of $2^{24}$ ) and put these primes into a file. Then for each odd $n$ with $2^{k-1}<n<2^{k}$, we were able to determine $\omega(n)$ and $\nu(n)$, using this file and trial division. These computations were done on a SPARC I computer and took only several hours to run.

Table III shows the values of $p_{k, 1}$ (approximated to six place accuracy) obtained by using the above equations in our program for $2 \leq k \leq 24$. Since all these values are less than $1 / 5$ we can conclude that $p_{k, t} \leq 4^{-t}$ for all $k$ with $2 \leq k \leq 24$ and $t \geq 1$. Combining these results with the results obtained using the upper bounds, we have shown that $p_{k, t} \leq 4^{-t}$ for all $k \geq 2$ and $t \geq 1$.

TABLE III. Computed values of $p_{k, 1}$ for $2 \leq k \leq 24$

\begin{tabular}{c|c||c|c||c|c||c|c}
$k$ & $p_{k, 1}$ & $k$ & $p_{k, 1}$ & $k$ & $p_{k, 1}$ & $k$ & $p_{k, 1}$ \\
\hline 2 & 0.000000 & 8 & 0.038004 & 14 & 0.005934 & 20 & 0.000609 \\
3 & 0.000000 & 9 & 0.030837 & 15 & 0.003944 & 21 & 0.000402 \\
4 & 0.164179 & 10 & 0.020525 & 16 & 0.002626 & 22 & 0.000276 \\
5 & 0.064299 & 11 & 0.017394 & 17 & 0.001929 & 23 & 0.000188 \\
6 & 0.065348 & 12 & 0.010710 & 18 & 0.001258 & 24 & 0.000126 \\
7 & 0.056655 & 13 & 0.007949 & 19 & 0.000905 & &
\end{tabular}




\section{Further NuMERICAL RESUlts}

Using the upper bound $p_{k, t} \leq N_{1} /\left(N_{1}+P_{1}\right)$ where $N_{1}$ and $P_{1}$ are defined as in $\S 3$, we can improve most of the values found in Table 2 of [3]. For each possible $k$ and $t$ in the table, we computed a lower bound for $-\lg p_{k, t}$ in the following way. For each $M$ within a well chosen range, we chose our $s$ as before to be the minimum of the largest allowable value for $s$ and 30 to obtain a lower bound for $-\lg p_{k, t}$ and we then took the maximum of all these lower bounds. The entries in Table IV reflect the maximum of the computed values and the entries from Table 2 of [3] where the italicized entries are those entries from [3] which were not improved upon by our computations. We believe that it is possible to improve this table even more by using the combined method discussed in [3] and the results obtained in this paper, but we did not attempt to do so.

TABLE IV. Lower bounds for $-\lg p_{k, t}$

\begin{tabular}{c|cccccccccc}
$k \backslash t$ & 1 & 2 & 3 & 4 & 5 & 6 & 7 & 8 & 9 & 10 \\
\hline 100 & 7 & 17 & 23 & 28 & 32 & 35 & 38 & 41 & 44 & 46 \\
150 & 11 & 22 & 30 & 36 & 41 & 46 & 50 & 53 & 56 & 60 \\
200 & 14 & 27 & 36 & 43 & 49 & 54 & 59 & 63 & 67 & 71 \\
250 & 16 & 32 & 42 & 49 & 56 & 62 & 68 & 72 & 77 & 81 \\
300 & 19 & 36 & 46 & 55 & 63 & 69 & 75 & 81 & 86 & 90 \\
350 & 28 & 39 & 51 & 60 & 69 & 76 & 82 & 88 & 94 & 99 \\
400 & 37 & 46 & 55 & 65 & 74 & 82 & 89 & 95 & 101 & 107 \\
450 & 46 & 54 & 62 & 70 & 79 & 88 & 95 & 102 & 108 & 114 \\
500 & 56 & 63 & 70 & 78 & 85 & 93 & 101 & 108 & 115 & 121 \\
550 & 65 & 72 & 79 & 86 & 93 & 100 & 107 & 114 & 121 & 128 \\
600 & 75 & 82 & 88 & 95 & 102 & 108 & 115 & 121 & 128 & 135
\end{tabular}

\section{ACKNOWLEDGMENT}

Special thanks go to Carl Pomerance who was instrumental in all aspects of writing this paper and without whom this paper could not have been written. The comments of Andrew Granville were also very much appreciated.

\section{REFERENCES}

1. P. Beauchemin, G. Brassard, C. Crépeau, and C. Goutier, Two observations on probabilistic primality testing, Advances in Cryptology-Crypto 86 Proceedings (A.M. Odlyzko, ed.), Lecture Notes in Comput. Sci., vol. 263, Springer-Verlag, Berlin, 1987, pp. 443-450. MR 89c: 11180

2. P. Beauchemin, G. Brassard, C. Crépeau, C. Goutier, and C. Pomerance, The generation of random numbers that are probably prime, J. Cryptology 1 (1988), 53-64. MR 89g:11126

3. I. Damgård, P. Landrock, and C. Pomerance, Average case error estimates for the strong probable prime test, Math. Comp. 61 (1993), 177-194. MR 94b:11124

4. L. Monier, Evaluation and comparison of two efficient probabilistic primality testing algorithms, Theoret. Comput. Sci. 12 (1980), 97-108. MR 82a:68078

5. M. O.Rabin, Probabilistic algorithm for testing primality, J. Number Theory 12 (1980), 128138. MR 81f: 10003

Department of Mathematics, University of Georgia, Athens, Georgia 30602-7403

E-mail address: ronnie@alpha.math.uga.edu 